\begin{tabular}{|c|}
\hline $\begin{array}{r}\text { PRAMANA } \\
\text { - journal of } \\
\text { physics }\end{array}$ \\
\hline
\end{tabular}

\title{
SuSeFLAV: A program for calculating supersymmetric spectra and lepton flavour violation
}

\author{
DEBTOSH CHOWDHURY, RAGHUVEER GARANI* and \\ SUDHIR K VEMPATI \\ Centre For High Energy Physics, Indian Institute of Science, Bangalore 560 012, India \\ ${ }^{*}$ Corresponding author. E-mail: rgarani@ cts.iisc.ernet.in
}

\begin{abstract}
The program SuSeFLAV is introduced for computing supersymmetric mass spectra with flavour violation in various supersymmetric breaking scenarios with/without see-saw mechanism. A short user guide summarizing the compilation, executables and the input files is provided.
\end{abstract}

Keywords. Minimal supersymmetric Standard Model; right-handed neutrinos; lepton flavour violation

PACS Nos 11.30.Pb; 12.15.Ff; 12.60.Jv; 13.35.Bv; 13.35.Dx; 14.60.Pq; 14.60.St

Presently, low-energy supersymmetry is facing its stiffest challenge in terms of direct experimental searches at LHC and indirect searches through flavour violating and dark matter experiments. It is thus important to have a complete program which takes into account flavour violation in the Yukawa couplings as well as in the soft sector. The presence of flavour violation through see-saw or otherwise can significantly modify the regions of parameter space which are compatible with experimental results. SuSeFLAV [1] is designed to consider flavour violation in both the Yukawa couplings as well as mass matrix computations. The short summary of the program is given below.

The numerical procedure implemented in the program can be summarized in a pictorial form presented in figure 1 .

Step 1: Low-energy inputs $M_{\mathrm{t}}^{\text {pole }}, M_{\mathrm{b}}^{\overline{\mathrm{MS}}}(\mathrm{mb}), M_{\tau}^{\text {pole }}, g_{1,2,3}^{\overline{\mathrm{DR}}}$ are defined in $\overline{\mathrm{DR}}$ scheme. The CKM matrix is also one of the inputs at the weak scale.

Step 2: The renormalization group equations (RGE) of the Yukawas and the gauge couplings are first evolved from the weak scale to the heaviest right-handed neutrino scale $\left(M_{\mathrm{R}_{3}}\right)$ where neutrino Dirac Yukawa $Y_{v}$ is defined. The RGE are then evolved to GUT scale, i.e., the scale at which $g_{1}=g_{2}$. If the right-handed neutrinos are switched off, the program directly checks for the GUT scale.

Step 2a: The default choices for the $Y_{v}$ are CKM-like mixing $\left(Y_{v}=V_{\mathrm{CKM}} Y_{\mathrm{U}}\right)$, PMNSlike mixing $\left(Y_{v}=U_{\mathrm{PMNS}} Y_{\mathrm{U}}\right)$ in the up-type Yukawa mass matrix $\left(Y_{\mathrm{U}}\right)$ at the 


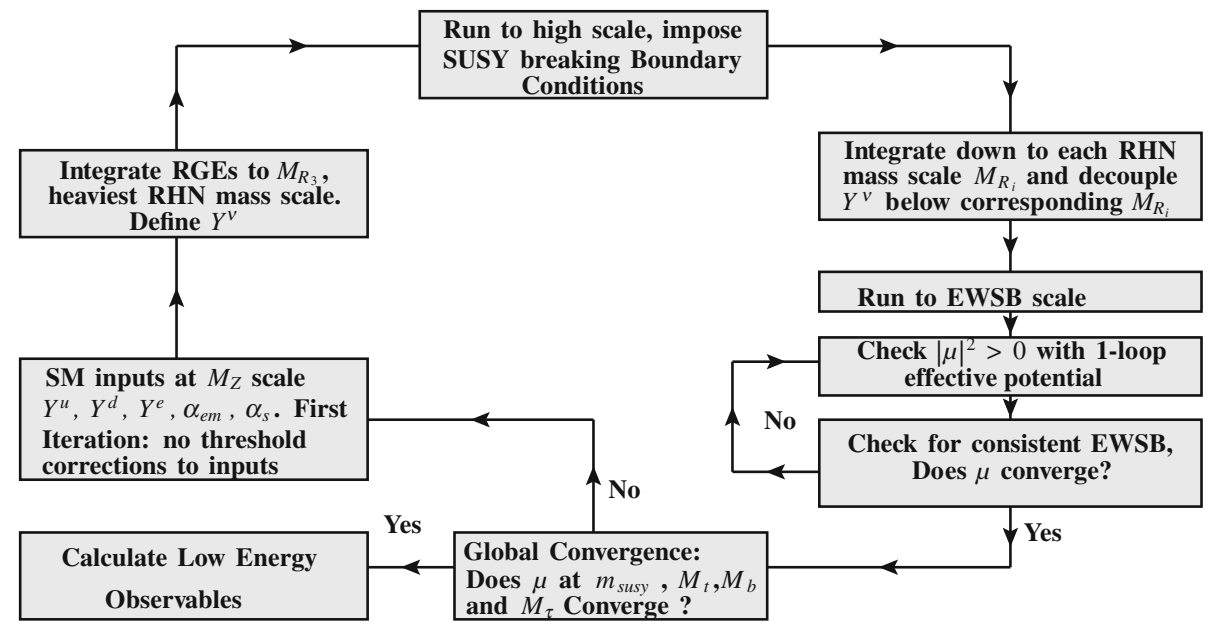

Figure 1. Flowchart of SUSEFLAV program.

see-saw scale. The user is also free to define his/her choice of the $Y_{v}$ either directly or by using the $R$-parametrization of Casas and Ibarra [2].

Step 3: At the GUT scale we impose boundary conditions corresponding to mSUGRA or non-universal Higgs masses (NUHM) or non-universal gaugino masses (NUGM) to all soft terms and run the corresponding RGE down to $M_{\mathrm{SUSY}}$, which is chosen to be $1 \mathrm{TeV}$ for the first iteration and the geometric mean of stop masses in the subsequent iterations. A completely non-universal (CNUM) set of boundary conditions can also be provided by the user. This choice is typically useful while studying models with broken flavour symmetries at high scale.

Step 3a: For the GMSB case, the program evolves only up to the messenger scale which is user-defined, instead of GUT scale as in other models.

Step 4: The program checks the radiative electroweak symmetry breaking conditions at $M_{\text {SUSY }}$. Corrections from the 1-loop effective potential are also taken into account. They can significantly modify the $\mu$ parameter, which in turn modifies the mass spectrum. An iterative procedure is employed to find solutions consistent with the mass spectrum as well as electroweak symmetry breaking conditions.

Step 5: One-loop supersymmetric threshold corrections to $M_{\mathrm{t}}\left(M_{\mathrm{Z}}\right), M_{\mathrm{b}}\left(M_{\mathrm{Z}}\right), M_{\tau}\left(M_{\mathrm{Z}}\right)$, $g_{1,2,3}\left(M_{\mathbf{Z}}\right)$ and $\sin ^{2} \theta_{w}$ are computed using the particle mass spectrum at $M_{\mathbf{Z}}$. These form the initial values of the Yukawa and the gauge couplings to the RGEs at the weak scale.

Step 6: The complete program is then put in an iterative cycle till particle masses at $M_{\mathrm{SUSY}}$ and $M_{\mathrm{t}}\left(M_{\mathrm{Z}}\right), M_{\mathrm{b}}\left(M_{\mathrm{Z}}\right)$ and $M_{\tau}\left(M_{\mathrm{Z}}\right)$ converge to the desired accuracy.

Step 7: Once the spectrum converges we compute all the low-energy observables mentioned like $(g-2)_{\mu}$ and lepton flavour violating observables like $\mu \rightarrow e+\gamma$ etc. 
SuSEFLAV requires LAPACK [3] library as a prerequisite for compilation. Running the provided Makefile would compile the package. This has been tested on LINUX operating systems and MAC OS-X. The package produces three executables when compiled, namely suseflav, suseflavslha and suseflavscan, to facilitate three different functionalities for its users. To compute the spectrum for a single point the usage of executables suseflav and suseflavslha is recommended, whereas, to scan the parameter space the usage of the executable suseflavscan can be used. SuSeFLAV has two input/output modes. These can be broadly classified into SLHA2 [4] I/O interface and non-SLHA or traditional SuSEFLAV I/O interface. Input files starting with sinputs should be used to run the program using the traditional SuSeFLAV interface. Each of these files provides an option to run right-handed neutrinos with user-defined mixing along with algorithm-specific inputs such as the precision of computation. Similarly, input files starting with slha should be used to run the program using the SLHA interface. The following is a list of input files which is provided with the program. All these files can be easily modified by the user to suit her/his purposes.

(1) sinputs.in/slha. in: These files contain the input parameters to run cMSSM or mSUGRA models.

(2) sinputs-nuhm. in/slha-nuhm. in: These files contain the input parameters to run models with non-universal Higgs masses in mSUGRA.

(3) sinputs-nugm. in: Input file to run models with non-universal gaugino masses in mSUGRA.

(4) sinputs-gmsb. in/slha-gmsb. in: Input files to run gauge-mediated supersymmetric breaking models.

(5) sinputs-rpar.in: Input file contains the parameters to run Casas-Ibarra $R$ parametrization [2] of neutrino masses and mixing with constrained MSSM boundary conditions.

(6) sinputs-cnum.in: This is the most generic input file for gravity-mediated models. All the SUSY breaking parameters at the high scale are defined by the user. This is useful for studying flavour models.

(7) sinputs_scan.in: This input file should be used to scan the parameter space of mSUGRA or NUHM.

SuSEFLAV can be coupled to dark matter routines such as MICROMEGAS, DarkSUSY and SuperIso to compute the relic density and direct detection rates. The program is free under the GNU Public License and can be downloaded from the following websites: (a) http://cts.iisc.ernet.in/Suseflav/main.html and (b) http://projects. hepforge.org/suseflav/.

\section{References}

[1] D Chowdhury, R Garani and S K Vempati, arXiv:1109.3551 [hep-ph]

[2] J A Casas and A Ibarra, Nucl. Phys. B618, 171 (2001), hep-ph/0103065

[3] http://www.netlib.org/lapack/

[4] B C Allanach, C Balazs, G Belanger, M Bernhardt, F Boudjema, D Choudhury, K Desch, U Ellwanger et al, Comput. Phys. Commun. 180, 8 (2009), arXiv:0801.0045 [hep-ph] 